\title{
The relationship between bladder wall thickness and lower urinary tract symptoms: Does bladder wall thickness change after alpha- blocker therapy with alfuzosin?
}

\author{
Ayhan Karakose, MD; Ozgu Aydogdu, MD; Yusuf Ziya Atesci, MD
}

Department of Urology, Izmir University, Izmir, Turkey

Cite as: Can Urol Assoc J 2014;8(1-2):e26-9. http://dx.doi.org/10.5489/cuaj.1513

Published online January 14, 2014.

\section{Abstract}

Introduction: We evaluate the association between lower urinary tract symptoms (LUTS) and bladder wall thickness (BWT) and investigate whether alfuzosin might improve BWT.

Methods: We retrospectively reviewed the data of 164 patients with LUTS. Patients were divided into 2 groups according to BWT (Group 1: BWT $\leq 5 \mathrm{~mm}, \mathrm{n}=69$; Group 2: BWT $>5 \mathrm{~mm}, \mathrm{n}=95$ ). Age, international prostate symptom score (IPSS), maximum and average urinary flow rates (Qmax and Qave), quality of life (QoL), postvoid residual (PVR) urine volume, prostate volume and prostate-specific antigen (PSA) were compared between the 2 groups. In total, 102 patients underwent transurethral resection of the prostate (TURP) and 62 patients were treated with alfuzosin. We compared BWT, Qmax, Qave, IPSS, QoL, PVR and PSA before and the sixth month of alfuzosin therapy. A $p$ value of $<0.05$ was considered statistically significant.

Results: The mean BWT of Group 1 was $3.72 \pm 0.56 \mathrm{~mm}$ and Group 2 was $6.43 \pm 1.13 \mathrm{~mm}$. There was a significant difference between the 2 groups in terms of mean Qmax and PVR. There was no statistical difference between the groups in terms of Qave, IPSS, QoL, prostate volume and PSA. There was significant difference between BWT before $(6.8 \pm 2.1)$ and after $(4.6 \pm 1.3)$ treatment with alfuzosin in 62 patients $(p=0.02)$. There was a significant difference between pre- and post-treatment values of mean Qmax, Qave, IPSS, QoL score, and PVR with alfuzosin.

Conclusion: BWT is a non-invasive and effective test to evaluate patients with lower urinary tract obstruction and may be used for showing the effectiveness of alpha-blocker therapy in patients with LUTS.

\section{Introduction}

Benign prostatic hyperplasia $(\mathrm{BPH})$ is common in men over 50 and causes lower urinary tract symptoms (LUTS).
Additionally, about $28 \%$ of men with $\mathrm{BPH}$ have moderate to severe LUTS. Bladder outlet obstruction (BOO) is seen in $52 \%$ of asymptomatic patients and in $60 \%$ of symptomatic patients. ${ }^{1-3}$ Studies have demonstrated that BOO is associated with changes in detrusor structure caused by smooth muscle hypertrophy, fibroelastic hyperplasia, and collagen deposition in the bladder wall. ${ }^{4}$

Bladder compliance decreases in humans and animals due to lower urinary tract obstruction, which is caused by increased collagen deposition in the bladder wall. Increased intravesical pressure results from smooth muscle hypertrophy and thus the bladder wall thickens. ${ }^{5,6}$ Alpha-blocker therapy is the most preferred medical treatment in patients with LUTS. 7,8

Bladder wall hypertrophy is caused by a thickening of the detrusor. Bladder wall thickness (BWT) is measured by ultrasound due to increasing obstruction grade in patients with benign prostatic obstruction. ${ }^{9,10}$ In this study we evaluate the relationship between LUTS and bladder wall thickness and investigate if alpha-blocker treatment with alfuzosin improves BWT.

\section{Methods}

We retrospectively analyzed the data of 164 patients admitted to the urology outpatient clinic with LUTS. We excluded patients with suspected cancer on digital rectal examination (DRE), prostate-specific antigen (PSA) $>4 \mathrm{ng} / \mathrm{mL}$, urinary tract infection (UTI), acute or chronic prostatitis, neurogenic bladder, urinary retention, a history of 5 alpha-reductase inhibitors, antimuscarinics or alpha-blocker therapy, bladder tumour, urolithiazis, diabetes mellitus and history of transurethral prostate surgery or lower urinary tract operation.

Hyperechogenic outer and inner layers of the bladder wall are visible on ultrasound and show the adventitia and mucosa/submucosal tissue. The hypoechogenic detrusor is visible and between the hyperechogenic lines of the adventitia and 
mucosa. The 3 layers show BWT and the detrusor measurement shows detrusor wall thickness (DWT). BWT decreases due to bladder filling. Oelke and colleagues showed that DWT decreases rapidly between 50 and $250 \mathrm{~mL}$ of bladder filling (or until $50 \%$ of bladder capacity), but reaches a plateau thereafter with only minor and insignificant differences between $250 \mathrm{~mL}$ and maximum bladder capacity. ${ }^{10}$ Therefore, we chose a bladder filling volume of $250 \mathrm{~mL}$. We filled the bladder via catheter to $250 \mathrm{~mL}$ and we evaluated each patient's BWT before and after treatment. BWT was measured by the same urologist before and after treatment with patients in the supine position using a suprapubic ultrasound (Philips HD7 ultrasound system, Netherlands ) anterior bladder wall with a $7.5-\mathrm{MHz}$ probe. We used $5 \mathrm{~mm}$ as a cut-off value for BWT based on a previous study by Manieri and colleagues. ${ }^{11}$ Patients were divided into 2 groups according to BWT values (Group 1: BWT $\leq 5 \mathrm{~mm}, \mathrm{n}=69$; Group 2: BWT $>5 \mathrm{~mm}, \mathrm{n}=95$ ). Age, international prostate symptom score (IPSS), maximum and average urinary flow rates (Qmax and Qave), quality of life (QoL) score, post-voiding residual urine volume (PVR), prostate volume and PSA levels were compared between 2 groups.

In total, 102 patients underwent transurethral resection of the prostate (TURP): 40 from Group 1 and 62 from Group 2 . After evaluating the IPSS, Qmax, Qave, QoL score, PVR, and prostate volume, we determined the surgical or medical treatment. Patients' preferences also affected the decision. The remaining 62 patients were treated with alfuzosin: 29 from Group 1 and 33 from Group 2. All patients were evaluated after 6 months of treatment. We compared the following values before treatment and after 6 months of treatment: BWT, Qmax, Qave, IPSS, QoL, PVR and PSA levels.

Clinical and laboratory data were analyzed with SPSS version 18.0. Data were displayed as mean \pm standard deviation (SD). Statistical evaluations were performed with Mann-Whitney $U$ test. A 5\% level of significance was used for all statistical testing. A $p$ value $<0.05$ was considered statistically significant.

\begin{tabular}{|c|c|c|c|}
\hline & BWT $\leq 5 \mathrm{~mm}(\mathrm{n}=69)$ & BWT >5 mm (n=95) & $p$ value \\
\hline Age (year) & $61.1 \pm 6.7$ & $62.4 \pm 7.6$ & 0.65 \\
\hline $\operatorname{Omax}(\mathrm{mL} / \mathrm{s})$ & $13.6 \pm 4.7$ & $9.8 \pm 5,7$ & 0.03 \\
\hline Qave (mL/s) & $4.6 \pm 0.5$ & $3.2 \pm 0.3$ & 0.51 \\
\hline IPSS score & $19.3 \pm 2.5$ & $21.6 \pm 2.1$ & 0.56 \\
\hline QoL score & $3.4 \pm 0.4$ & $3.7 \pm 0.6$ & 0.76 \\
\hline $\begin{array}{l}\text { Prostate } \\
\text { volume }(\mathrm{mL})\end{array}$ & $55.3 \pm 14.5$ & $57.8 \pm 16.7$ & 0.72 \\
\hline PVR (mL) & $113.7 \pm 50.8$ & $137.2 \pm 60.4$ & 0.03 \\
\hline PSA (ng/dL) & $2.3 \pm 1.2$ & $2.4 \pm 1.1$ & 0.83 \\
\hline
\end{tabular}

BWT: bladder wall thickness; LUTS: lower urinary tract symptoms; Omax: maximum flow rate; Qave: average flow rate; PVR: postvoid residual; PSA: prostate-specific antigen; OoL: quality of life; IPSS: International Prostate Symptom Score.

\section{Results}

The mean age of all patients $(n=164)$ was $63.2 \pm 8.1$ years (range: 43-75). The mean age of patients in Group 1 and Group 2 were $61.1 \pm 6.7$ and $62.4 \pm 7.6$ years, respectively. There was no difference between the groups in terms of mean age $(p=0.65)$. The mean BWT was $5.25 \pm 1.76 \mathrm{~mm}$ in all patients. The mean BWT of patients in Group 1 and Group 2 were $3.72 \pm 0.56 \mathrm{~mm}$ and $6.43 \pm 1.13 \mathrm{~mm}$, respectively. There was significant difference between the 2 groups in terms of mean Qmax $(p=0.03)$ and PVR $(p=0.03)$. There was no statistical difference between the 2 groups in terms of Qave, IPSS, QoL score, prostate volume, and PSA (Table 1).

Of the 164 patients, 102 underwent TURP and 62 were treated with alfuzosin. The mean age of the patients treated with alfuzosin was $61.7 \pm 5.1$ (range: $43-69$ ) years. The BWT decreased in $47(75.8 \%)$ patients after treatment. There was a significant difference between pre- and post-treatment BWT measurements with alfuzosin $(6.8 \pm 2.1$ and $4.6 \pm 1.3 \mathrm{~mm}$, respectively) $(p=0.02)$. There was a significant difference between pre- and post-treatment values with alfuzosin in terms of mean Qmax $(p=0.01)$, Qave $(p=0.04)$, IPSS $(p=0.02)$, QoL score $(p=0.03)$, and PVR $(p=0.01)$, respectively. There was no statistical difference in terms of PSA values $(p=0.87)$ (Table 2$)$.

\section{Discussion}

The main reason for LUTS is BPH in adult men. Partial obstruction due to BPH causes epithelial and fibroelastic hyperplasia in bladder smooth muscle and mucosa. This obstruction increases BWT and decreases the contraction and membranous functions of the bladder. Previous studies demonstrated significant changes in the extracellular matrix structure of the obstructed bladder and increases in the collagen type 3/type 1 ratio. This causes proliferation in mucosa, submucosa, detrusor, and serosa and a decrease in bladder compliance affecting the response of the bladder to filling and contraction. ${ }^{12-14}$

\begin{tabular}{|c|c|c|c|}
\hline & Pre-treatment & Post-treatment & $p$ value \\
\hline BWT $(\mathrm{mm})$ & $6.8 \pm 2.1$ & $4.6 \pm 1.3$ & 0.02 \\
\hline $\operatorname{Omax}(\mathrm{mL} / \mathrm{s})$ & $10.8 \pm 3.4$ & $15.2 \pm 2.7$ & 0.01 \\
\hline Qave (mL/s) & $3.8 \pm 0.9$ & $7.6 \pm 1.8$ & 0.04 \\
\hline IPSS score & $19.7 \pm 4.6$ & $13.9 \pm 3.7$ & 0.02 \\
\hline QoL score & $3.6 \pm 0.5$ & $2.3 \pm 0.4$ & 0.03 \\
\hline PVR (mL) & $126.3 \pm 55.4$ & $76.4 \pm 32.3$ & 0.01 \\
\hline PSA (ng/dL) & $2.1 \pm 1.1$ & $2.2 \pm 0.9$ & 0.87 \\
\hline
\end{tabular}

BWT: bladder wall thickness; LUTS: lower urinary tract symptoms; Omax: maximum flow rate; Qave: average flow rate; PVR: postvoid residual; PSA: prostate-specific antigen; QoL: quality of life; IPSS: International Prostate Symptom Score. 
Although urodynamic examination is the gold standard to evaluate LUTS, its invasive nature limits its use. Previous studies advocated that BWT could be used to evaluate lower urinary tract obstruction. Oelke and colleagues performed a urodynamic evaluation in patients with BPH and/or LUTS. They found that BWT was more valuable compared to uroflowmetry, PVR, and prostate volume in the evaluation of LUTS. ${ }^{6}$ Manieri and colleagues investigated the data of patients who were clinically diagnosed with $\mathrm{BPH}$ via urodynamic evaluation. They found a strong association between urodynamic pressure parameters and BWT; and a weak association between urodynamic pressure parameters and Qmax and PVR. ${ }^{11}$ Hakanberg and colleagues showed a weak positive association between age and BWT in patients with LUTS and diagnosed as BPH. ${ }^{15}$ In another study, researchers found that BWT was higher in patients with a Qmax $<10 \mathrm{~mL} / \mathrm{s}$ and no significant relationship between BWT and IPSS. ${ }^{16}$ Our findings demonstrated a mean BWT of $5.25 \pm 1.76$ in all patients. The mean BWT of Group 1 was $3.72 \pm 0.56$ and Group 2 was $6.43 \pm 1.13$. There was a significant difference between the 2 groups in terms of mean $\operatorname{Qmax}(p=0.03)$ and PVR $(p=0.03)$. There was no statistical difference between the 2 groups in terms of mean age, Qave, IPSS, QoL score, prostate volume, and PSA.

Manieri and colleagues showed lower urinary tract obstruction with urodynamic investigation and found a cut-off value of $5 \mathrm{~mm}$ for BWT. The authors detected lower urinary tract obstruction in $87.5 \%$ of patients with LUTS and BWT $>5 \mathrm{~mm} .{ }^{11}$ Another study showed that BWT $\geq 2 \mathrm{~mm}$ is $95.5 \%$ diagnostic for lower urinary tract obstruction. ${ }^{15}$ Yilmaz and colleagues concluded that BWT measurement is critical to show the duration of lower urinary tract obstruction in patients with LUTS. ${ }^{17}$

Recent studies showed that BWT significantly correlated with parameters of pressure-flow study. Alpha-blocker treatment improves Qmax, Qave, IPSS, QoL score, and structural changes in bladder wall caused by lower urinary tract obstruction in patients with LUTS., ${ }^{218}$ Ultrasound studies showed that alpha-blocker treatment decreases PVR and increases of Qmax, which correlated with decreases in bladder wall hypertrophy. ${ }^{10}$ Alpha-blocker treatment with tamsulosin in men with prostatic hyperplasia decreased the ultrasound-estimated bladder weight (from 65 to $28 \mathrm{~g}$ ). ${ }^{13,14}$ In another study, the authors found that BWT was associated with intravesical prostatic protrusion and worsening storage symptom scores in BPH patients with LUTS. They found that alpha-blocker therapy did not improve irritative symptoms in BPH patients and that their BWT increased. The authors concluded that BWT may be useful to predict responsiveness of irritative symptoms to alpha-blocker treatment in BPH patients with LUTS. ${ }^{18}$ In another study, improvements in voiding symptoms and decreases in BWT after tamsulosin treatment indicated that ultrasound measurements of BWT, with flow rate studies and IPSS, could be considered an outcome assessment tool in the pharmacologic treatment of $\mathrm{BPH} .{ }^{2}$ In the present study, we found a significant difference between BWT measured before and 6 months after alpha-blocker treatment with alfuzosin. There was a significant difference between pre- and post-treatment values with alfuzosin in terms of mean Qmax, Qave, IPSS, QoL score, and PVR.

Although our study suggests a non-invasive test to clinically evaluate patients with LUTS, it has some limitations. The main limitation of this study is its retrospective nature. We only investigated patients who were treated with alphablocker therapy; patients who underwent TURP were not included in the study. All BWT measurements were performed when the bladder filling volume was $250 \mathrm{~mL}$. BWT is affected by gender, aging, and pathological states, such as BPH and bladder volume. ${ }^{10,15}$ Our findings would probably be more valuable if the BWT measurements were done for a standardized bladder volume. In addition, we only used alfuzosin for alpha-blocker therapy and did not investigate the potential effects of other alpha-blockers. All BWT measurements were performed with suprapubic ultrasound probe and we did not compare our findings with transrectal ultrasound probe measurements.

\section{Conclusion}

BWT measurement with suprapubic ultrasound probe represents a non-invasive and effective test to evaluate patients with lower urinary tract obstruction. BWT measurement may also be used as a potential test to show the effectiveness of alpha-blocker therapy in patients with LUTS.

Competing interests: Dr. Karakose, Dr. Aydogdu and Dr. Atesci all declare no competing financial or personal interests.

This paper has been peer-reviewed.

\section{References}

1. Barsch G, Müller HR, Oberholzer $M$, et al. Light microscobic stereological analysis of the normal human prostate and of benign prostatic hyperplasia. J Urol 1994;122:487-91.

2. Eghbali K, Shayegan MR, Kianoush S. Investigating the effect of tamsulosin on the measurement of bladder wall thickness and International Prostate Symptom Score in benign prostatic hyperplasia. Can Urol Assoc J 2013;7 (5-6):317-21.

3. Hutchison A, Farmer R, Chapple C, et al. Characteristics of patients presenting with LUTS/BPH in six European countries. Eur Urol 2006;50:555-62. http://dx.doi.org/10.1016/j.eururo.2006.05.001

4. Levin RM, Haugaard N, $O^{\prime}$ Connor L, et al. Obstructive response of human bladder to BPH vs. rabbit bladder response to partial outlet obstruction: A direct comparison. Neurourol Urodyn 2000;19:609-29. http:// dx.doi.org/10.1002/1520-6777(2000) 19:5<609::AID-NAU7>3.0.C0;2-H

5. Matsumoto $S$, Chichester $P$, Kogan $B A$, et al. Structural and vascular response of normal and obstructed rabbit whole bladders to distension. Urology 2003;62:1129-33. http://dx.doi.org/10.1016/S00904295(03)00686-1 
6. Oelke $M$, Höfner $K$, Jonas $U$, et al. Diagnostic accuracy of noninvasive tests to evaluate bladder outlet obstruction in men: Detrusor wall thickness, uroflowmetry, postvoid residual urine, and prostate volume. Eur Urol 2007;52:827-34. http://dx.doi.org/10.1016/i.eururo.2006.12.023

7. Oelke M, Bachmann A, Descazeaud A, et al. EAU Guidelines on the Management of Male Lower Urinary Tract Symptoms (LUTS), including Benign Prostatic Obstruction (BP0); 2013. http://www.uroweb. org/fileadmin/guidelines/Total_file_2013_large_guidelines_prints.pdf. Accessed December 12, 2014.

8. McVary KT, Roehrborn CG, Avins AL, et al. Update on AUA guideline on the management of benign prostatic hyperplasia. J Urol 2011;185:1793-803. http://dx.doi.org/10.1016/i.juro.2011.01.074

9. Oelke $M$, Höfner $K$, Wiese B, et al. Increase in detrusor wall thickness indicates bladder outlet obstruction (BO0) in men. World J Urol 2002;19:443-52.

10. Oelke M. International Consultation on Incontinence-Research Society (ICI-RS) report on non-invasive urodynamics: The need of standardization of ultrasound bladder and detrusor wall thickness measurements to quantify bladder wall hypertrophy. Neurourol Urodyn 2010;29:634-9. http://dx.doi.org/10.1002/ nau.20834

11. Manieri C, Carter SS, Romano G, et al. The diagnosis of bladder outlet obstruction in men by ultrasound measurement of bladder Wall thickness. J Urol 1998;159:761-5. http://dx.doi.org/10.1016/S0022$5347(01) 63723-6$

12. Levin RM, Levin SS, Zhao Y, et al. Cellular and molecular aspects of bladder hypertrophy. Eur Urol 1997; $32: 15-21$.

13. Egilmez T, Pourbagher MA, Guvel $S$, et al. Effects of selective alpha-1-adrenergic receptor blockers on bladder weight. Urol Int 2006;76:42-50. http://dx.doi.org/10.1159/000089734
14. Milicevic $S$. Tamsulosin efficiency in treatment of benign prostatic hyperplasia evaluated by determining bladder weight. Med Arh 2012;66:391-5. http://dx.doi.org/10.5455/medarh.2012.66.391-395

15. Hakenberg $\mathrm{OW}$, Linne $C$, Manseck $A$, et al. Bladder wall thickness in normal adults and men with mild lower urinary tract symptoms and benign prostatic enlargement. Neurourol Urodyn 2000;19:585-93. http://dx.doi.org/10.1002/1520-6777(2000) 19:5<585::AID-NAU5>3.0.C0;2-U

16. Işikay L, Turgay Akgül K, et al. Lower urinary tract symptoms, prostate volume, uroflowmetry, residual urine volume and bladder Wall thickness in Turkish men: A comparative analysis. Int Urol Nephrol 2007;39:1131-5. http://dx.doi.org/10.1007/s11255-006-9161-y

17. Yılmaz A, Aslan Y, Uzun B, et al. Tuncel A, Atan A. Relationship between bladder wall thickness and duration of symptoms, uroflowmetry parameters, and international prostate symptom score in patients with lower urinary tract symptoms. Turk J Urol 2009;35:361-5.

18. Park JS, Lee HW, Lee SW, et al. Bladder wall thickness is associated with responsiveness of storage symptoms to alpha-blockers in men with lower urinary tract symptoms. Korean I Urol 2012;53:487-91. htrp://dx.doi.org/10.4111/kju.2012.53.7.487

Correspondence: Dr. Ayhan Karakose, Department of Urology, Izmir University, Izmir, Turkey; drayhankarakose@gmail.com 